\title{
The scope of tobacco cessation randomized controlled trials in low- to middle-income countries: protocol for a scoping review
}

\author{
Navin Kumar $^{1 *}$, Jessica Ainooson ${ }^{1}$, Ameera Billings ${ }^{1}$, Grace Chen $^{1}$, Lauren Cueto ${ }^{1}$, Kamila Janmohamed ${ }^{1}$, \\ Jeannette Jiang ${ }^{1}$, Raymond Niaura ${ }^{2,3}$ and Amy Zhang ${ }^{1}$
}

\begin{abstract}
Background: Tobacco consumption is the leading cause of preventable death globally. The global mortality burden of tobacco use lies predominantly in low- to middle-income countries (LMICs). There is much evidence on the effectiveness of tobacco cessation RCTs in high-income nations. However, the evidence base in LMICs is far more limited. To effectively design randomized controlled trials (RCTs) that mitigate tobacco-related harms in LMICs, further understanding of RCTs in this environment will be helpful. We will provide quality evidence regarding the scope of tobacco cessation RCTs in LMICs.

Methods: A scoping review of tobacco cessation RCTs will be conducted. MEDLINE, Embase, PsycINFO, Global Health, Web of Science and Sociological Abstracts will be searched to capture current literature. We will review RCTs that have already been done on tobacco cessation in the LMICs. The key outcome will be tobacco cessation in adults. Examples of the key outcome include smoking abstinence and reduction of tobacco use. Study selection will conform to Preferred Reporting Items for Systematic Reviews and Meta-Analyses Extension for Scoping Reviews (PRISMA-ScR) guidelines and study quality assessed with a modified version of the Cochrane Collaboration's instrument.

Discussion: As researchers attempt to minimize the harms from tobacco in LMICS, they need to be aware of scientific evidence to develop RCTs to achieve their aim. The review will complement the evidence base on tobacco cessation in LMICS.
\end{abstract}

Keywords: Tobacco, Low- to middle-income countries, Cessation, Abstinence

\section{Background}

Tobacco consumption is the leading cause of preventable death globally $[1,2]$. Most of global mortality burden of tobacco use lies predominantly in low- to middle-income countries [3]. Low- to middle-income countries (LMICs) are experiencing a growing epidemic of tobacco use [4].

\footnotetext{
* Correspondence: Navin.kumar@yale.edu

${ }^{1}$ Human Nature Lab, Department of Sociology, Yale University, New Haven, $C T$, USA

Full list of author information is available at the end of the article
}

Tobacco use rates have been increasing in LMICs $[5,6]$. Tobacco control is key to any nation's public health strategy $[7,8]$. Tobacco control, such as cessation interventions, should thus be a priority for policymakers in LMICs to mitigate effects of tobacco-related morbidity and mortality $[1,2]$.

There are stark differences between LMICs and highincome nations regarding the proportion of smokers who want to quit $[9,10]$. Intention to quit smoking in high-income nations is about $75 \%[11,12]$, while LMICs 
still lag far behind. For example, $41 \%$ of Indian smokers and smokeless tobacco users did not want to quit [13]. In high-income nations, prevalence has significantly declined [14]. For example, Australia has seen a 50\% decrease in smoking prevalence from $26.1 \%$ in 1991 to $13.3 \%$ in 2013 [15]. LMIC smoking rates still persist [16].

Previous reviews explored tobacco cessation in LMICs [17-24]. However, these reviews were limited as they did not include studies across all LMICs nor centered on randomized controlled trials (RCTs). There are multiple observational and quasi-experimental studies on tobacco cessation in LMICs [25-27]. However, tobacco cessation RCTs are minimal in this context. While we acknowledge the issues inherent with RCTs [28-30], we also indicate that other study designs are not an adequate replacement for RCTs in establishing efficacy [31], which is key in tobacco cessation [32, 33].

The main objective of this scoping review was to locate and review all published literature relating to tobacco cessation RCTs in LMICs, detailing gaps in the literature. For example, we will detail if there are LMICs where comparatively fewer RCTs have been conducted. We chose to conduct a scoping review due to the broad research question, suited for mapping an area of research [34]. While scoping reviews normally include all evidence, not just RCTs, [35] we sought to focus on RCTs for two reasons. Firstly, previous reviews had centered on other study designs, such as quasi experimental studies. Secondly, to effectively design RCTs that mitigate tobacco-related harms in LMICs, further understanding of RCTs in this environment will be helpful. Research in this area may augment knowledge about tobacco products, broaden understanding around tobacco use among diverse populations, and improve understanding of tobacco firm strategies [36]. Greater understanding around tobacco cessation research may also aid the evidence base to enhance tobacco cessation scholarship, policy, and implementation globally, mitigating the tobacco epidemic [36]. In this scoping review, we will evaluate evidence on the scope of tobacco cessation RCTs, already conducted, in LMICs.

This review will expand on past literature to detail methodological and scientific progress of previously conducted tobacco cessation RCTs in LMICs. We will follow the Preferred Reporting Items for Systematic Reviews and Meta-Analyses Extension for Scoping Reviews (PRISMA-ScR) guidelines, use standard tools to assess study quality $[37,38]$ and propose a reproducible strategy to query the literature about the scope of tobacco cessation RCTs in LMICs.

\section{Methods/design}

Search strategy

The search strategy will be performed in line with techniques that enhance methodological transparency and improve the reproducibility of the results and evidence synthesis. Thus, the search strategy will be elaborated and implemented prior to study selection, with the PRISMA-ScR checklist as guidance [37]. We will use the following guiding question to ensure a scoping literature search: "What is the scope of tobacco cessation RCTs in LMICs?". We will review RCTs already conducted on tobacco cessation in LMICs.

Studies will be reviewed across six databases, including MEDLINE, Embase, PsycINFO, Global Health, Web of Science, and Sociological Abstracts. To account for contemporary studies, a literature search will be conducted till September 2020. No language restrictions will be imposed. Reference lists of the articles will be used to identify more studies. We will also conduct a gray literature search using Google Scholar, clinical trials registries, and governmental websites. We will speak with leading tobacco control experts to identify any relevant studies. EndNote, a bibliographic software, will be used to store, organize, and manage all references [39]. Covidence will be used to manage the title/abstract and full-text screening phases [40]. We will use the search strategy indicated in Additional file 1 . We will manually exclude non-RCTs to avoid bias.

\section{Study selection criteria}

Studies will be excluded if they were conducted in the high-income nations. LMICs and high-income nations were defined based on the World Bank's per capita gross national income metric [41] (see Additional file 2). Two independent reviewers will screen each title and abstract as per inclusion/exclusion criteria (see below). Only studies involving adults ( $\geq 18$ years) were included. The key outcome will be tobacco cessation. Examples of the key outcome include smoking abstinence and reduction of tobacco use.

\section{Inclusion criteria}

- Research was conducted in LMICs

- Research investigating tobacco cessation in adults

- Randomized controlled trials (RCTs)

\section{Exclusion criteria}

- Any commentaries, editorials, or opinion pieces

- Research conducted in high-income countries

- Qualitative studies

- Non-RCT studies

- Studies involving only children or adolescents

\section{Study selection}

Reviewers will be trained in calibration and utilized standardized screening forms. Reviewers will work in teams 
of two and independently screen all titles and abstracts that we identify by the literature search strategy. We will obtain full-text articles of all possibly eligible studies and evaluate article eligibility. Reviewers will resolve disagreement around eligibility by discussion or, if necessary, with a third reviewer. Studies reported only as conference abstracts will also be included and we will cite all articles utilizing data from such studies. Conference abstracts are often left out of systematic reviews as they may not contain adequate information [42]. We will include conference abstracts as they are more likely to contain positive results and are often published sooner [42], key to a scoping review on RCTs. We will contact authors where necessary if the abstracts do not provide sufficient information [42].

\section{Data extraction}

Four pairs of reviewers will undergo practice exercises and then work in pairs to independently extract data from studies. Reviewers will resolve disagreement through discussion. When differences are unable to be resolved, a third reviewer will make the final decision. Reviewers will abstract the data using a pretested data extraction template, which includes study design, participants, interventions, comparators, and outcomes.

\section{Risk of bias assessment}

While it is not common to assess risk of bias in scoping reviews [43], we are only including RCTs, and thus, the review is amenable for study quality assessment. Reviewers will work in pairs to independently assess the risk of bias for included RCTs. Disagreements will be resolved by a third reviewer. We will use a modified version of the Cochrane Collaboration's instrument [44]. The instrument includes nine domains: adequacy of sequence generation, allocation sequence concealment, participant blinding, data collectors blinding, outcome assessment blinding, data analyst blinding, incomplete outcome data, selective outcome reporting, and potential sources of bias. We will contact study authors for additional information when information regarding risk of bias or other methodological aspects is unavailable. The risk of bias will be summarized as a narrative statement and supported by a risk of bias table.

\section{Descriptive analysis}

A narrative synthesis of the outcomes of selected studies will be detailed in the final review. We will include information such as: type of intervention, sample size, target population and demographic characteristics, intervention outcomes.

\section{Amendments}

Any amendments to this protocol will be documented with reference to saved searches and analysis.

\section{Dissemination}

Results of the review will be disseminated in a peerreviewed journal and likely in other media such as: conferences, seminars, symposia.

\section{Discussion}

The strength of the planned study is the use of a transparent and reproducible procedure for a scoping literature review. In this protocol, we detailed types of studies, participants, interventions, and outcomes included. We stated the data sources, search strategy, data extraction, and quality assessment methods [45]. Through publishing the research protocol, we strengthen the clarity of the search strategy and reduce risk of bias, such as selective outcome reporting [46]. Moreover, we center solely on the scope of tobacco cessation RCTs in LMICs. Results will thus provide high-level information to inform, support, and customize design of RCTs in this setting. As researchers attempt to minimize the harms from tobacco in LMICs, they need to be aware of scientific evidence to develop RCTs to achieve their aim. The planned study hopes to build knowledge around inadvertent outcomes of tobacco cessation interventions and enhance understanding around tobacco control advocacy efforts [36].

\section{Supplementary information}

Supplementary information accompanies this paper at https://doi.org/10. 1186/s13643-020-01361-2.

Additional file 1. Medline search example.

Additional file 2. Definitions of Low- to middle-income countries (LMICS) and High-Income Nations.

\section{Abbreviations}

RCT: Randomized controlled trial; PRISMA-ScR: Preferred Reporting Items for Systematic Reviews and Meta-Analyses Extension for Scoping Reviews; LMIC: Low- to middle-income countries

\section{Acknowledgements}

The authors thank the reviewers, editors, and Melissa Funaro for her assistance.

\section{Authors' contributions}

All authors contributed to the manuscript and read and approved the final draft.

\section{Funding}

Study was funded by the Foundation for a Smoke-Free World. The funding body had no role in the design, analysis, or interpretation of the data in the study.

Availability of data and materials

The datasets used and analyzed are available from the corresponding author on reasonable request.

Ethics approval and consent to participate Not applicable

Consent for publication Not applicable 


\section{Competing interests}

The authors declare that they have no competing interests.

\section{Author details}

${ }^{1}$ Human Nature Lab, Department of Sociology, Yale University, New Haven, CT, USA. ${ }^{2}$ Department of Social and Behavioral Sciences, College of Global Public Health, New York University, 715 Broadway, New York, NY, USA. ${ }^{3}$ Department of Epidemiology, College of Global Public Health, New York University, 715 Broadway, New York, NY, USA.

\section{Received: 22 November 2019 Accepted: 14 April 2020}

\section{Published online: 21 April 2020}

\section{References}

1. Ghebreyesus TA. Progress in beating the tobacco epidemic. Lancet. 2019; 394:548-9.

2. Agaku IT, Alpert HR. Trends in annual sales and current use of cigarettes, cigars, roll-your-own tobacco, pipes, and smokeless tobacco among US adults, 2002-2012. Tob Control. 2016;25:451-7.

3. Sinha DN, Suliankatchi RA, Gupta PC, Thamarangsi T, Agarwal N, Parascandola $\mathrm{M}$, et al. Global burden of all-cause and cause-specific mortality due to smokeless tobacco use: systematic review and metaanalysis. Tob Control. 2018;27:35-42

4. Sreeramareddy $C T$, Harper S, Ernstsen L. Educational and wealth inequalities in tobacco use among men and women in 54 low-income and middleincome countries. Tob Control. 2018:27:26-34.

5. Jawad M, Lee JT, Millett C. Waterpipe tobacco smoking prevalence and correlates in 25 eastern Mediterranean and Eastern European countries: cross-sectional analysis of the Global Youth Tobacco Survey. Nicotine Tob Res. 2016;18:395-402.

6. Sinha DN, Gupta PC, Kumar A, Bhartiya D, Agarwal N, Sharma S, et al. The poorest of poor suffer the greatest burden from smokeless tobacco use: a study from 140 countries. Nicotine Tob Res. 2018;20:1529-32.

7. Schreuders M, Lagerweij NA, van den Putte B, Kunst AE. To what extent and why adolescents do or do not support future tobacco control measures: a multimethod study in the Netherlands. Tobacco Control. 2017;27:596-9. https://doi.org/10.1136/tobaccocontrol-2017-053770.

8. Goodchild M, Zheng R. Tobacco control and Healthy China 2030. Tob Control. 2018;28:409-13. https://doi.org/10.1136/tobaccocontrol-2018054372

9. Kulak JA, Cornelius ME, Fong GT, Giovino GA. Differences in quit attempts and cigarette smoking abstinence between Whites and African Americans in the United States: literature review and results from the International Tobacco Control US Survey. Nicotine Tob Res. 2016;18(Suppl 1):S79-87.

10. Saqib MAN, Rafique I, Qureshi H, Munir MA, Bashir R, Arif BW, et al. Burden of tobacco in Pakistan: findings from Global Adult Tobacco Survey 2014. Nicotine Tob Res. 2019;21:136.

11. Marques-Vidal P, Melich-Cerveira J, Paccaud F, Waeber G, Vollenweider P, Cornuz J. Prevalence and factors associated with difficulty and intention to quit smoking in Switzerland.. BMC Public Health 2011;11:227.

12. Disease Control $C$ for, (CDC $P$, others. Current cigarette smoking among adults-United States, 2011.. MMWR Morbidity and mortality weekly report 2012; 61:889.

13. Singh PK, Yadav A, Lal P, Sinha DN, Gupta PC, Swasticharan L, et al. Dual burden of smoked and smokeless tobacco use in India 2009-2017: a repeated cross-sectional analysis based on Global Adult Tobacco Survey. Nicotine Tob Res 2020. doi:https://doi.org/10.1093/ntr/ntaa033.

14. Feliu A, Filippidis FT, Joossens L, Fong GT, Vardavas Cl, Baena A, et al. Impact of tobacco control policies on smoking prevalence and quit ratios in 27 European Union countries from 2006 to 2014. Tobacco Control 2018; tobaccocontrol-2017-054119. doi:https://doi.org/10.1136/tobaccocontrol2017-054119.

15. Health Al of, Welfare. National Drug Strategy Household Survey Detailed Report 2013. supplementary tobacco smoking table S3. 2. Drug Statistics Series No. 28. Cat. No. PHE 1832014

16. Hughes N, Arora M, Grills N. Perceptions and impact of plain packaging of tobacco products in low and middle income countries, middle to upper income countries and low-income settings in high-income countries: a systematic review of the literature. BMJ Open. 2016;6:e010391.

17. Sinha DN, Palipudi KM, Rolle I, Asma S, Rinchen S. Tobacco use among youth and adults in member countries of South-East Asia region: review of findings from surveys under the Global Tobacco Surveillance System. Indian J Public Health. 2011;55:169-76.

18. Brathwaite R, Addo J, Smeeth L, Lock K. A systematic review of tobacco smoking prevalence and description of tobacco control strategies in SubSaharan African countries; 2007 to 2014. PLoS One. 2015;10:e0132401.

19. Nagarkar SAGA. Tobacco control interventions during last decade in India: a narrative review. National Journal of Community Medicine 2017.

20. Alzahrane A, West R, Ubhi HK, Brown J, Abdulqader N, Samarkandi O. Evaluations of clinical tobacco cessation interventions in Arab populations: a systematic review. Addict Behav. 2019;88:169-74.

21. Blecher $E$. The impact of tobacco advertising bans on consumption in developing countries. J Health Econ. 2008:27:930-42.

22. Abdelwahab SI, Yagoub U, Sunosi RA, Bulgiba A, Hatim A, Alahmar A. Smoking cessation efforts in special population: a review of research on Muslim countries and communities. Int J Pharmacol. 2013;9:98-107. https:// doi.org/10.3923/ijp.2013.98.107.

23. Kristina SA, Thavorncharoensap M. Smoking and smoking cessation in Indonesia and other Muslim countries: a systematic review. Res Soc Administr Pharm. 2012;8:e29. https://doi.org/10.1016/j.sapharm.2012.08.068.

24. Kim SS, Chen W, Kolodziej M, Wang X, Wang VJ, Ziedonis D. A systematic review of smoking cessation intervention studies in China. Nicotine Tob Res. 2012;14:891-9. https://doi.org/10.1093/ntr/ntr289.

25. Chelwa G, van Walbeek C, Blecher E. Evaluating South Africa's tobacco control policy using a synthetic control method. Tob Control. 2016;26:50917. https://doi.org/10.1136/tobaccocontrol-2016-053011.

26. Goel S, Sharma D, Gupta R, Mahajan V. Compliance with smoke-free legislation and smoking behaviour: observational field study from Punjab India. Tob Control. 2017;27:407-13. https://doi.org/10.1136/tobaccocontrol-2016-053559.

27. Schensul JJ, Nair S, Bilgi S, Cromley E, Kadam V, Mello SD, et al. Availability accessibility and promotion of smokeless tobacco in a low-income area of Mumbai. Tob Control. 2012;22:324-30. https://doi.org/10.1136/ tobaccocontrol-2011-050148.

28. Carey TA, Stiles WB. Some problems with randomized controlled trials and some viable alternatives. Clin Psychol Psychother. 2015;23:87-95. https://doi. org/10.1002/cpp.1942

29. McCambridge J, Kypri K, Elbourne D. In randomization we trust? There are overlooked problems in experimenting with people in behavioral intervention trials. J Clin Epidemiol 2014;67:247-253. doi:https://doi.org/10. 1016/j.jclinepi.2013.09.004.

30. Bothwell LE, Greene JA, Podolsky SH, Jones DS. Assessing the gold standard lessons from the history of RCTs. N Engl J Med. 2016;374:2175-81. https:// doi.org/10.1056/nejmms1604593.

31. Gerstein HC, McMurray J, Holman RR. Real-world studies no substitute for RCTs in establishing efficacy. Lancet. 2019;393:210-1. https://doi.org/10. 1016/s0140-6736(18)32840-x.

32. Heckman CJ, Egleston BL, Hofmann MT. Efficacy of motivational interviewing for smoking cessation: a systematic review and meta-analysis. Tob Control. 2010;19:410-6. https://doi.org/10.1136/tc.2009.033175.

33. Zhu S-H, Lee M, Zhuang Y-L, Gamst A, Wolfson T. Interventions to increase smoking cessation at the population level: how much progress has been made in the last two decades? Tob Control. 2012;21:110-8. https://doi.org/ 10.1136/tobaccocontrol-2011-050371.

34. Arksey H, OMalley L. Scoping studies: towards a methodological framework. Int J Soc Res Methodol. 2005;8:19-32. https://doi.org/10.1080/ 1364557032000119616.

35. Pham MT, Rajić A, Greig JD, Sargeant JM, Papadopoulos A, McEwen SA. A scoping review of scoping reviews: advancing the approach and enhancing the consistency. Res Synth Methods. 2014;5:371-85.

36. Berg CJ, Fong GT, Thrasher JF, Cohen JE, Maziak W, Lando H, et al. The impact and relevance of tobacco control research in low-and middleincome countries globally and to the US. Addict Behav. 2018;87:162-8.

37. McGowan J, Straus S, Moher D, Langlois EV, O'Brien KK, Horsley T, et al. Reporting scoping reviews - PRISMA ScR extension. J Clin Epidemiol 2020.

38. Guyatt GH, Busse JW. Modification of Cochrane tool to assess risk of bias in randomized trials. Distiller http://distillercer.com/resources/ (access: 201703 26) 2015.

39. Analytics C. Endnote X8 for Windows. Philadelphia, PA: Clarivate Analytics 2017

40. Innovation VH. Covidence systematic review software; 2017.

41. Bank W. World Bank Country and Lending Groups - World Bank Data Help Desk. 2019. https://datahelpdesk.worldbank.org/knowledgebase/ articles/906519. 
42. Scherer RW, Saldanha IJ. How should systematic reviewers handle conference abstracts? A view from the trenches. Syst Rev. 2019;8:264.

43. Munn Z, Peters MDJ, Stern C, Tufanaru C, McArthur A, Aromataris E. Systematic review or scoping review? Guidance for authors when choosing between a systematic or scoping review approach. BMC Medical Research Methodology 2018;18. doi:https://doi.org/10.1186/s12874-018-0611-x.

44. Higgins JPT, Altman DG, Gotzsche PC, Juni P, Moher D, Oxman AD, et al. The Cochrane Collaborations tool for assessing risk of bias in randomised trials. BMJ 2011; 343 oct18 2:d5928-d5928. doi:https://doi.org/10.1136/bmj. d5928.

45. Silagy CA, Middleton P, Hopewell S. Publishing protocols of systematic reviews. JAMA. 2002;287:2831. https://doi.org/10.1001/jama.287.21.2831.

46. Green S, Higgins JPT. Preparing a Cochrane review. In: Cochrane Handbook for Systematic Reviews of Interventions. John Wiley \& Sons Ltd. 2008. p. 1130. https://doi.org/10.1002/9780470712184.ch2.

\section{Publisher's Note}

Springer Nature remains neutral with regard to jurisdictional claims in published maps and institutional affiliations.

Ready to submit your research? Choose BMC and benefit from:

- fast, convenient online submission

- thorough peer review by experienced researchers in your field

- rapid publication on acceptance

- support for research data, including large and complex data types

- gold Open Access which fosters wider collaboration and increased citations

- maximum visibility for your research: over $100 \mathrm{M}$ website views per year

At BMC, research is always in progress.

Learn more biomedcentral.com/submissions 